\title{
Pitavastatin calcium improves endothelial function and delays the progress of atherosclerosis in patients with hypercholesterolemia*
}

\author{
Jing ZHAO ${ }^{1,2}$, Hui-min YAN ${ }^{1,3}$, Ya LI $^{1,2}$, Jia WANG ${ }^{1,2}$, Lu HAN $^{1,2}$, Zhi-hao WANG ${ }^{1,4}$, \\ Meng-xiong TANG ${ }^{1,5}$, Wei ZHANG ${ }^{1,2}$, Yun ZHANG ${ }^{1,2}$, Ming ZHONG ${ }^{\dagger 1,2}$ \\ ( ${ }^{1}$ Key Laboratory of Cardiovascular Remodeling and Function Research, Chinese Ministry of Education and Chinese Ministry of Public Health, \\ Qilu Hospital of Shandong University, Ji'nan 250012, China) \\ ('Department of Cardiology, Qilu Hospital of Shandong University, Ji'nan 250012, China) \\ ( ${ }^{3}$ Department of Cardiology, Longfu Hospital of Dongcheng District, Beijing 100010, China) \\ $\left({ }^{4}\right.$ Department of Geriatrics, Qilu Hospital of Shandong University, Ji'nan 250012, China) \\ ( ${ }^{5}$ Department of Emergency, Qilu Hospital of Shandong University, Ji'nan 250012, China) \\ †E-mail: zhongmingzm@gmail.com
}

Received June 30, 2014; Revision accepted Jan. 27, 2015; Crosschecked Apr. 9, 2015

\begin{abstract}
Background: Statins have proven efficacy in inhibiting the onset and progress of atherosclerosis. The effectiveness of pitavastatin in reversing carotid atherosclerosis associated with hypercholesterolemia (HC) is unknown. Objectives: To explore the simultaneous effects of pitavastatin calcium on brachial arterial flow-mediated vasodilatation (FMD), carotid intima-media thickness (IMT), and arterial stiffness $(\beta)$, three surrogate markers of atherosclerosis were studied in $\mathrm{HC}$ patients. Methods: A randomized, double-blind trial was performed with $40 \mathrm{HC}$ subjects who fulfilled the inclusion/exclusion criteria. Patients were given pitavastatin calcium $1 \mathrm{mg} / \mathrm{d}$ (Group 1) or $2 \mathrm{mg} / \mathrm{d}$ (Group 2) for 8 weeks. There were 20 patients in each group, and 30 gender- and age-matched healthy subjects as controls were recruited. FMD of the brachial artery, carotid IMT, and arterial stiffness indicated by $\beta$ were measured at baseline and at 8 weeks after starting pitavastatin calcium therapy using ultrasound techniques. Biochemical tests were also made on all subjects. Results: At baseline, higher total cholesterol (TC) and low-density lipoprotein cholesterol (LDL-C), reduced FMD, and increased $\beta$ and IMT were observed in HC patients $(P<0.001$ for all) compared with controls. After 8 weeks, TC was decreased by $20.59 \% / 27.56 \%$ and LDL-C $30.92 \% / 35.64 \%$, respectively, in comparison to baseline groups; the $\mathrm{HC}$ groups had reduced $\beta$ and improved endothelial function over the 8-week follow-up $(P<0.05-0.001)$; nonetheless, no significant alterations of IMT were found $(P>0.05)$. Significant negative interactions between TC/LDL and FMD $(P<0.05-0.001)$, positive interactions between TC and IMT $(P=0.003)$ and between TC/LDL and $\beta(P<0.001-0.000)$ were found. Conclusions: Treatment with pitavastatin calcium exerted favorable effects on endothelial function and arterial stiffness. It also improved carotid atherosclerosis in patients with $\mathrm{HC}$.
\end{abstract}

Key words: Flow-mediated vasodilatation (FMD), Hypercholesterolemia (HC), Carotid intima-media thickness (IMT), Pitavastatin calcium

doi: $10.1631 /$ jzus.B1400181

Document code: A

CLC number: R589.2

\section{Introduction}

Large-scale epidemiological studies have demon-

ॠ Corresponding author

* Project supported by the National Science and Technology Major Project (No. 2012ZX09303-016-003) and the National Natural Science Foundation of China (Nos. 81270352, 81270287, 81300168, 81471036 , and 81470560)

(DD ORCID: Ming ZHONG, http://orcid.org/0000-0003-0514-6028

(C) Zhejiang University and Springer-Verlag Berlin Heidelberg 2015 strated that hypercholesterolemia (HC) is a vital risk factor for atherosclerosis (AS) and the subsequent development of cardiovascular events (Galle et al., 1990). Impaired flow-mediated vasodilatation (FMD), increased arterial stiffness $(\beta)$, and carotid intima- 
media thickness (IMT) reflect early AS (functionally and structurally) and have been found to predict cardiovascular events (Lorenz et al., 2007; Yeboah et al., 2007; Leone et al., 2008; Yu et al., 2014). Brachial FMD response is correlated with coronary endothelial function as tested by invasive methods (Anderson et al., 1995; Takase et al., 1998) and impaired FMD parallels the extent of coronary AS (Neunteufl et al., 1997). Endothelial dysfunction (ED) is an early event in AS, which precedes structural changes in the vascular wall (Ross, 1993). IMT represents a marker of structural AS, and increased IMT is correlated with the severity of coronary AS (Burke et al., 1995). Therefore, it is important to assess the severity of AS in all its aspects, both functionally and structurally, in $\mathrm{HC}$ patients.

Statins have been proved to be extremely effective in reducing cardiovascular risk, as demonstrated in clinical studies confirming the inhibition of the onset and progress of AS. These benefits attribute to its pleiotropy, including modulation of endothelial function, alleviation of arterial stiffness, and stabilization of plaque (Cho et al., 2009). Pitavastatin calcium has been described as a "super statin" with stronger low-density lipoprotein cholesterol (LDL-C)/ triglyceride (TG)-lowering and high-density lipoprotein (HDL)-elevating effects compared with other statins at the initial dose. The evidence regarding the efficacy of pitavastatin on reversing carotid AS associated with $\mathrm{HC}$ is unknown, while ED has been shown to improve (as in our previous study on pitavastatin (Hiro et al., 2009)), while administration of pitavastatin in patients with acute coronary syndrome (ACS) has been shown to cause regression of coronary plaques. Based on these findings, the present study was aimed to investigate the impact of pitavastatin calcium on functional and structural markers of early AS in $\mathrm{HC}$ patients.

\section{Materials and methods}

\subsection{Subjects}

Patients with $\mathrm{HC}$ were recruited from the Qilu Hospital of Shandong University (Ji'nan, China) according to the following inclusion/exclusion criteria. Inclusion criteria: (1) age, 18-70 years; (2) total cholesterol (TC), 5.72-12.70 mmol/L and/or LDL-C,
3.64-6.50 mmol/L; (3) TG, $<4.52 \mathrm{mmol} / \mathrm{L}$. Exclusion criteria: (1) severe heart failure and arrhythmia; (2) thyroid disease diabetes (fasting blood glucose (FBG), $\geq 10 \mathrm{mmol} / \mathrm{L}$ ); (3) severe liver or kidney disease; (4) any lipid-lowering medication (e.g. statins or ezetimibe) taken 2 weeks before the study. Thirty healthy subjects (mean age, $(54.94 \pm 6.90)$ years; 14 men and 16 women) matched for age and sex were recruited as controls. The protocol was approved by the Human Research Committees of Qilu Hospital, and all patients gave written informed consent.

\subsection{Protocol}

The 40 patients in the present study included those subjects who had undergone at least 2 weeks of a wash-out period and met the inclusion/exclusion criteria. They were then randomly divided into two groups: Group $1(1 \mathrm{mg} / \mathrm{d}$ pitavastatin calcium, $5 \mathrm{men}$ and 15 women $)$ and Group $2(2 \mathrm{mg} / \mathrm{d}$ pitavastatin calcium, 9 men and 11 women). By the end of the study, 2 patients were lost in each group. All the patients were examined before and 8 weeks after pitavastatin therapy. Pitavastatin calcium was kindly provided by Ji'nan Limin Pharmaceutical Co., Ltd., China.

\subsection{Laboratory examination}

Venous blood was withdrawn from all subjects for measurement of serum TC, LDL-C, TG, HDL-C, and FBG after a fasting period of 10-12 h. All biochemical indices were measured using a Hitachi-76 Fully-automatic Biochemical Analyzer (Shizuokaken, Japan).

\subsection{Brachial flow-mediated artery vasodilatation}

Brachial FMD was assessed following the guidelines of the International Brachial Artery Reactivity Task Force (Corretti et al., 2002) using high-resolution ultrasound (Acuson Sequoia 512 with a $13.0 \mathrm{MHz}$, linear-array transducer). The brachial artery was scanned $5-10 \mathrm{~cm}$ above the elbow. Brachial artery diameters were measured at baseline $\left(D_{0}\right)$ and 5 min after forearm hyperemia $\left(D_{1}\right)$, produced by releasing a forearm cuff inflated to $280-300 \mathrm{mmHg}$ for $5 \mathrm{~min}$. The change in vessel diameter was immediately expressed as a percentage relative to the baseline vessel diameter before cuff inflation (FMD). The formula used was $\mathrm{FMD} \%=\left(D_{1}-D_{0}\right) / D_{0} \times 100 \%$. 
The diameters of three cardiac cycles were analyzed for each scan, and the measurements were averaged. Throughout the study, FMD was measured by the same skilled examiner, who used the same ultrasound apparatus and probe set. We have previously reported inter-study coefficient of variation (CV) of $9 \%$ for FMD measurements (Cho et al., 2009).

\subsection{Carotid artery ultrasound}

Carotid ultrasound studies were performed as previously described (Raitakari et al., 2003). In brief, subjects were examined in a supine position with the head turned to the opposite direction at $45^{\circ}$. Left and right arteries were viewed at end diastole/systole (the top of the R and $\mathrm{T}$ waves) in transverse and longitudinal projections. The carotid diameter was measured at least three times in end-diastole $\left(D_{\mathrm{d}}\right)$ and endsystole $\left(D_{\mathrm{s}}\right)$, respectively. IMT was evaluated as the distance between the lumen-intima interface and the media-adventitia interface, and free of any discrete plaques. The measurements of IMT were taken at the far wall of three segments of bilateral carotid arteries: the bifurcation (the $1.0 \mathrm{~cm}$ segment proximal to the flow divider), the distal common carotid artery (the $1.0 \mathrm{~cm}$ segment proximal to the dilatation of the carotid bulb), and the proximal common carotid artery (the $1.0 \mathrm{~cm}$ segment proximal to the descending section of common carotid artery) to derive mean IMT. All measurements were made offline from stored digital images.

Ultrasound and concomitant brachial blood pressure measurements were used to calculate carotid stiffness $(\beta): \beta=\frac{\ln (\mathrm{SBP} / \mathrm{DBP})}{\Delta D / D_{\mathrm{d}}}$, where $\Delta D$ is the difference between systolic and diastolic diameters, and SBP and DBP are systolic and diastolic blood pressures (Makita et al., 2000). The intra- and interobserver coefficients of variation for IMT measurements were $4.3 \%$ and $4.4 \%$, respectively.

\subsection{Statistical analyses}

Data are presented as means \pm standard deviation (SD) unless otherwise stated. Continuous variables were compared between groups by an independent $t$-test or paired Student's $t$-test. Bivariate correlations underwent partial correlation analysis to assess possible relationship between serum lipids and FMD, and $\beta$ and IMT after adjusting covariates. Next, multiple linear regression analysis was constructed to identify significant determinants of FMD, $\beta$, and IMT. The analysis of covariance (ANCOVA) was used to analyze the effects of pitavastatin calcium on atherosclerosis. A $P$ value of $<0.05$ was considered statistically significant. Analyses used SPSS v.16.0 (SPSS Inc., Chicago, IL, USA).

\section{Results}

\subsection{Comparison of clinical and biochemical characteristics of the subjects}

The characteristics of patients currently receiving pitavastatin calcium compared with the controls are shown in Table 1. These data are part of our previous research (Yan et al., 2011). Patients with HC in Group $1(1 \mathrm{mg} / \mathrm{d})$ had significant higher body mass index (BMI), hip circumference, FBG, TC, TG, and LDL-C $(P<0.05-0.001)$ compared with the controls before pitavastatin calcium therapy. Patients with HC in Group $2(2 \mathrm{mg} / \mathrm{d})$ had significant higher BMI, hip circumference, SBP, DBP, FBG, TC, TG, and LDL-C $(P<0.05-0.001)$. After 8 weeks of pitavastatin therapy, TC and TG were lowered by $20.59 \%$ and $30.92 \%$ (6.41 to $5.09 \mathrm{mmol} / \mathrm{L}$ and 2.07 to $1.43 \mathrm{mmol} / \mathrm{L}$, $P<0.01-0.001$ ), respectively in Group 1 and $27.56 \%$ and $35.64 \%$ (6.75 to $4.89 \mathrm{mmol} / \mathrm{L}, 2.02$ to $1.30 \mathrm{mmol} / \mathrm{L}$, $P$ both $<0.001)$, respectively in Group 2 . No difference in any index was observed between Group 1 and Group 2 after therapy.

\subsection{Comparison of ultrasonic parameters}

The ultrasound parameters of patients and controls are also summarized in Table 1. When compared with controls, patients with HC (both Group 1 and Group 2) had significantly greater $\beta$ and IMT ( $P$ both $<0.001)$ and lower FMD $((6.05 \pm 0.82) \%$ vs. $(11.33 \pm$ $0.81) \%,(7.10 \pm 0.61) \%$ vs. $(11.33 \pm 0.81) \%$, respectively, $P$ both $<0.001)$ at baseline. It showed that the function and structure of the peripheral vascular system were impaired in $\mathrm{HC}$ before pitavastatin calcium therapy. After 8 weeks of pitavastatin therapy, FMD and $\beta$ were significantly improved compared with baseline values in Group 1 (9.62\% vs. $6.05 \%, 3.24$ vs. 3.72 , respectively, $P<0.05-0.001)$ and in Group 2 ( $9.50 \%$ vs. $7.10 \%$ and 3.34 vs. 3.62 , respectively, $P<0.05-0.01)$; however, no significant changes in IMT for both Group 1 and Group $2(P>0.05)$ were 
Table 1 Clinical characteristics, hematologic profile, and ultrasound indexes of the studied population

\begin{tabular}{|c|c|c|c|c|c|}
\hline \multirow{3}{*}{ Variable } & \multirow{3}{*}{$\begin{array}{l}\text { Control } \\
(n=30)\end{array}$} & \multicolumn{4}{|c|}{ Hypercholesterolemia } \\
\hline & & \multicolumn{2}{|c|}{ Group 1} & \multicolumn{2}{|c|}{ Group 2} \\
\hline & & $\begin{array}{c}\text { Pre-therapy } \\
(n=20)\end{array}$ & $\begin{array}{c}\text { Post-therapy } \\
(n=18)\end{array}$ & $\begin{array}{c}\text { Pre-therapy } \\
(n=20)\end{array}$ & $\begin{array}{c}\text { Post-therapy } \\
(n=18)\end{array}$ \\
\hline Age (year) & $54.94 \pm 6.90$ & $55.20 \pm 8.35$ & $56.00 \pm 7.85$ & $57.56 \pm 6.09$ & $57.79 \pm 6.46$ \\
\hline BMI $\left(\mathrm{kg} / \mathrm{m}^{2}\right)$ & $24.18 \pm 3.25$ & $26.65 \pm 2.78^{* *}$ & $26.90 \pm 2.88^{* *}$ & $26.43 \pm 3.52^{*}$ & $26.43 \pm 3.68^{*}$ \\
\hline $\mathrm{WC}(\mathrm{cm})$ & $86.48 \pm 7.71$ & $90.50 \pm 9.06$ & $90.67 \pm 11.07$ & $90.90 \pm 11.32$ & $90.81 \pm 10.71$ \\
\hline Hip circumference $(\mathrm{cm})$ & $100.05 \pm 6.87$ & $104.25 \pm 6.13^{*}$ & $105.28 \pm 5.96^{*}$ & $104.55 \pm 9.24^{*}$ & $105.39 \pm 9.52^{*}$ \\
\hline WHR & $0.86 \pm 0.09$ & $0.87 \pm 0.06$ & $0.86 \pm 0.07$ & $0.87 \pm 0.07$ & $0.86 \pm 0.06$ \\
\hline SBP (mmHg) & $123.87 \pm 11.86$ & $132.55 \pm 19.43$ & $129.72 \pm 18.51$ & $132.55 \pm 14.01^{*}$ & $131.64 \pm 17.21$ \\
\hline DBP (mmHg) & $82.00 \pm 8.48$ & $86.75 \pm 9.31$ & $89.11 \pm 13.00^{*}$ & $89.30 \pm 11.74^{*}$ & $90.08 \pm 13.26^{*}$ \\
\hline $\mathrm{TC}(\mathrm{mmol} / \mathrm{L})$ & $4.78 \pm 0.76$ & $6.41 \pm 0.51^{* * *}$ & $5.09 \pm 1.07^{* * \# \#}$ & $6.75 \pm 0.83^{* * *}$ & $4.89 \pm 0.78^{*+++}$ \\
\hline $\mathrm{TG}(\mathrm{mmol} / \mathrm{L})$ & $1.09 \pm 0.26$ & $2.07 \pm 0.78^{* * *}$ & $1.43 \pm 0.29^{* * \# \#}$ & $2.02 \pm 0.96^{* * *}$ & $1.30 \pm 0.51^{+++}$ \\
\hline HDL (mmol/L) & $1.40 \pm 0.34$ & $1.34 \pm 0.26$ & $1.44 \pm 0.25$ & $1.49 \pm 0.38$ & $1.56 \pm 0.31$ \\
\hline $\mathrm{LDL}(\mathrm{mmol} / \mathrm{L})$ & $2.51 \pm 0.47$ & $4.05 \pm 0.68^{* * *}$ & $2.85 \pm 0.86^{\# \# \#}$ & $4.13 \pm 0.51^{* * *}$ & $2.68 \pm 0.52^{+++}$ \\
\hline $\mathrm{FBG}(\mathrm{mmol} / \mathrm{L})$ & $5.06 \pm 0.50$ & $5.63 \pm 0.63^{* *}$ & $5.56 \pm 0.60^{*}$ & $5.49 \pm 0.58^{* *}$ & $5.60 \pm 0.44^{* * *}$ \\
\hline FMD (\%) & $11.33 \pm 0.81$ & $6.05 \pm 0.82^{* * *}$ & $9.62 \pm 0.93^{\#}$ & $7.10 \pm 0.61^{* * *}$ & $9.50 \pm 0.96^{+}$ \\
\hline$D_{\mathrm{d}}(\mathrm{mm})$ & $6.39 \pm 0.57$ & $6.56 \pm 0.49$ & $6.58 \pm 0.76^{\#}$ & $6.72 \pm 0.53^{*}$ & $6.62 \pm 0.76$ \\
\hline$D_{\mathrm{s}}(\mathrm{mm})$ & $5.97 \pm 0.56$ & $6.30 \pm 0.48^{*}$ & $6.20 \pm 0.72$ & $6.42 \pm 0.56^{* *}$ & $6.08 \pm 0.55^{++}$ \\
\hline$\beta$ & $3.14 \pm 0.19$ & $3.72 \pm 0.28^{* * *}$ & $3.24 \pm 0.13^{\# \# \#}$ & $3.62 \pm 0.18^{* * *}$ & $3.34 \pm 0.28^{* *++}$ \\
\hline IMT (mm) & $0.64 \pm 0.07$ & $0.78 \pm 0.05^{* * *}$ & $0.75 \pm 0.05^{* * *}$ & $0.76 \pm 0.04^{* * *}$ & $0.74 \pm 0.05^{* * *}$ \\
\hline $\mathrm{SR}_{\max }\left(\mathrm{s}^{-1}\right)$ & $38.48 \pm 6.45$ & $32.40 \pm 4.26^{* *}$ & $35.69 \pm 5.18^{\#}$ & $32.39 \pm 5.63^{* *}$ & $37.12 \pm 7.92^{+}$ \\
\hline $\mathrm{SR}_{\min }\left(\mathrm{s}^{-1}\right)$ & $18.35 \pm 3.00$ & $15.61 \pm 2.52^{* *}$ & $17.50 \pm 2.28^{\# \# \#}$ & $15.75 \pm 3.11^{* *}$ & $18.59 \pm 2.98^{+}$ \\
\hline
\end{tabular}

WHR: waist-hip ratio; $\mathrm{SR}_{\max }$ : strain rate maximum; $\mathrm{SR}_{\min }$ : strain rate minimum. ${ }^{*} P<0.05,{ }^{* *} P<0.01,{ }^{* * *} P<0.001$ vs. Control. ${ }^{*} P<0.05$,

${ }^{\#} P<0.01,{ }^{\#} P<0.001$ vs. $1 \mathrm{mg}$ pitavastatin pre-therapy group. ${ }^{+} P<0.05,{ }^{++} P<0.01,{ }^{+++} P<0.001$ vs. $2 \mathrm{mg}$ pitavastatin pre-therapy group

found. No difference in any index was noted between Group 1 and Group 2 after therapy.

\subsection{Correlations between FMD, $\beta$, IMT and var- ious risk variables}

Partial correlations of FMD, $\beta$, and IMT with various risk factor variables are presented in Table 2 . The inverse correlations between TC/LDL-C with FMD were significant $(P<0.05-0.001)$ after adjusting for age, weight, BMI, waist circumference (WC), waist-hip ratio (WHR), SBP, DBP, cigarette and alcohol consumption, and FBG. Simultaneously, the existence of positive correlations have been found between TC and IMT $(P=0.000)$ and between TC, LDL-C, and $\beta(P<0.001-0.000)$, when the same risk factors were controlled. The results demonstrate that $\mathrm{TC}$ and LDL-C are independent risk factors for impaired endothelial function and TC is also an independent risk factor for increased IMT in HC patients.

\subsection{Screening risk variables for increased carotid FMD, IMT, and $\beta$ by multiple linear regression analysis}

Stepwise multiple linear regression analysis was performed to identify significant determinants of
Table 2 Correlations between IMT, $\beta$, FMD and various factors

\begin{tabular}{clcc}
\hline Variable & Factor & $r$ & $P$ \\
\hline IMT & Weight & 0.219 & 0.024 \\
& BMI & 0.324 & 0.001 \\
& WC & 0.245 & 0.011 \\
& TC & 0.436 & 0.000 \\
& LDL & 0.288 & 0.003 \\
& TG & 0.239 & 0.015 \\
& FBG & 0.307 & 0.001 \\
& Smoke & 0.289 & 0.003 \\
& Drink & 0.261 & 0.007 \\
& Statins & -0.228 & 0.019 \\
\hline Weight & 0.223 & 0.021 \\
& BMI & 0.296 & 0.002 \\
& WC & 0.249 & 0.010 \\
& DBP & 0.220 & 0.023 \\
& TG & 0.494 & 0.000 \\
& TC & 0.395 & 0.000 \\
FMD & LDL & 0.479 & 0.000 \\
& Statins & -0.232 & 0.017 \\
\hline Statins & -0.232 & 0.017 \\
& BMI & -0.230 & 0.018 \\
& TC & -2.252 & 0.009 \\
& LDL & -0.327 & 0.001 \\
\hline & & &
\end{tabular}


FMD, $\beta$, and IMT in age, weight, BMI, WC, WHR, SBP, DBP, cigarette and alcohol consumption, FBG, and lipids (Table 3 ). Only BMI $(P=0.012)$ and LDL-C $(P=0.029)$ were risk factors for FMD; only BMI $(P=0.006)$, WC $(P=0.030)$, TG $(P=0.001)$, and LDL-C cholesterol $(P=0.001)$ were risk factors for $\beta$; and only BMI $(P=0.008)$, WC $(P=0.006)$, alcohol consumption $(P=0.045)$, and TC $(P=0.000)$ were risk factors for IMT.

Table 3 Relationships between FMD, $\beta$, IMT and various factors

\begin{tabular}{lclrc}
\hline Variable & Model & Factor & $r$ & $P$ \\
\hline IMT & 1 & BMI & 0.263 & 0.008 \\
& & Statins & -0.204 & 0.034 \\
& 2 & WC & 0.262 & 0.006 \\
& & Statins & -0.219 & 0.020 \\
& & Drink & 0.186 & 0.045 \\
& 3 & TC & 0.485 & 0.000 \\
\hline$\beta$ & 1 & BMI & 0.280 & 0.006 \\
& 2 & WC & 0.222 & 0.030 \\
& 3 & LDL & 0.315 & 0.001 \\
& & TG & 0.349 & 0.001 \\
\hline FMD & 1 & BMI & -1.210 & 0.012 \\
& 3 & LDL & -0.223 & 0.029 \\
\hline
\end{tabular}

Model 1 includes the following confounders: age, BMI, SBP, DBP, smoke, drink, statins. Model 2 includes the following confounders: age, WC, WHR, SBP, DBP, smoke, drink, statins. Model 3 includes the following confounders: lipids profile, FBG, alanine aminotransferase (ALT), aspartate transaminase (AST)

\subsection{Analysis of covariance}

Since even in patients with adequate reduction of the serum LDL-C levels, development of atherosclerotic disease, especially coronary artery disease (CAD), is not fully inhibited, risk factors other than LDL-C should also be considered (Barter et al., 2007; Dembowski and Davidson, 2009), including age, weight, BMI, WC, WHR, SBP, DBP, cigarette and alcohol consumption, and FBG. FMD and IMT were not significant after such risk factors were controlled ( $P>0.05$ for both), but $\beta$ was significantly decreased $(P<0.05)$. If lipid profiles were controlled, then FMD, $\beta$, and IMT were clearly improved ( $P<0.05$ for all); if lipid profiles and other risk factors were controlled simultaneously, FMD and $\beta$ were significantly improved $(P<0.05-0.001)$, but IMT showed no significance $(P>0.05)$. These results indicated that TC and LDL-C, but not the other risk factors, are the most important risk factors for ED and impaired function and structure of the carotid artery. Furthermore, treatment with pitavastatin calcium leads to an improvement in endothelial function and a prevention in carotid IMT in patients with HC.

\section{Discussion}

The main consequence of our study is that it shows a positive effect on vascular properties over an 8 -week period of pitavastatin therapy: lessening of $\mathrm{ED}$, decreased $\beta$, and decreased IMT progression. These indicate that early AS changes can be reversed by pitavastatin calcium therapy.

AS is the pathogenic process that underlies most cardiovascular diseases, particularly coronary heart disease (CHD) (Lusis, 2000), which is the main cause of increased morbidity and mortality (Weidner, 2000). A large body of evidence has shown that $\mathrm{HC}$ leads to AS by causing ED, which is a marker of cardiovascular (Anderson, 2006) and atherosclerotic (Bonetti et al., 2003) risk and responsible for the triggering of the first step of AS (Ross, 1993). Consistent with the idea that the TC and LDL are the major harmful factors for endothelial function, and the higher levels of TC/LDL, the greater the arterial stiffness (Diehm et al., 2004), we found that FMD, $\beta$, and IMT were still correlated significantly with TC after adjustment for the other risk factors. To the best of our knowledge, the data from our study provide for the first time evidence that pitavastatin calcium ameliorates endothelial function and attenuates arterial stiffness (Table 4). Another interesting finding was that increased carotid IMT (however, IMT $<0.90 \mathrm{~mm}$ (Zanchetti et al., 2002)) has still been found among $\mathrm{HC}$ patients without the other risk factors (Juonala et al., 2004) in the analysis of covariance (Table 4), suggesting that endothelial function was directly damaged by TC and LDL. This damage may then render arteries susceptible to the development of AS, e.g. by changing its permeability,

Table 4 Effects of pitavastatin calcium on endothelial function and arterial stiffness in hypercholesterolemia patients

\begin{tabular}{lcc}
\hline Variable & $F$ & $P$ \\
\hline$\beta$ & 3.127 & 0.036 \\
IMT & 2.506 & 0.047 \\
FMD & 2.993 & 0.022 \\
\hline
\end{tabular}


by increasing the adhesiveness of the endothelium to leukocytes, and by inducing endothelial expression of vasoactive molecules favoring arterial stiffening and increased IMT (Ross, 1999). Thus, reduced FMD identifies abnormalities of endothelial function preceding the development of a structural lesion, and IMT indicates the presence of vascular damage, suggestive of a more advanced stage of AS (Chen et al., 1995). It is essential to recognize early arterial changes in the both functional and structural changes terms, to inhibit progress of AS.

Several noninvasive ultrasound techniques have recently been developed to study FMD, carotid, $\beta$, and IMT. The close correlation between endothelial function of the brachial artery (detectable by FMD) and the endothelial function of the coronary artery (detectable by cardiac catheterization angiography) has been documented (Anderson et al., 1995). Decreased FMD probably contributes in part to the increase in arterial stiffness, and decreased FMD and increased arterial stiffness have been shown to be related to increased IMT in the Cardiovascular Risk in Young Finns (CRYF) study (Juonala et al., 2004). Increased IMT was demonstrated to be correlated closely with the severity of coronary AS (Bots et al., 1997), and IMT is meant to be used as an indicator for general AS including coronary AS (Juonala et al., 2004). Undoubtedly, both the endothelial functions of coronary artery and carotid artery can be assessed precisely by brachial FMD. It is likely that ED occurred as high cholesterol levels damage the endothelium, which is an important regulator of arterial stiffness, both functionally and structurally (Oliver and Webb, 2003). Structural changes may translate functionally into increased arterial stiffness and aggravated ED. Moreover, functional alteration of arterial tone, in conjunction with structural alteration of the arterial wall, contributes to the overall increase of arterial stiffness, generating a vicious circle which contributes to the pathogenesis of the atherosclerotic process and acceleration of the progression of AS. Thus, it is important to emphasize the preventivetherapeutic significance of the vascular consequence cascade.

Recent studies have been focused on the pleiotropic effects of statins on prevention and limitation of the development of AS, including the improvement of vascular endothelial function, anti-inflammatory actions, anti-oxidative effects, or stabilization of vulnerable plaques, which reduce the risk of cardiovascular events and cardiovascular mortality (Serruys et al., 2002). Previously our (Yan et al., 2011) and Petnehazy et al. (2005)'s studies have shown that ED can be improved in the $\mathrm{HC}$ patients by pitavastatin calcium therapy, which is considered as a "superstatin" because of its stronger lipid-lowering effect. At present, data on the clinical impact of pitavastatin on FMD and $\beta$ combined with IMT in the HC patient remain scarce. Our results demonstrated that the FMD was increased and $\beta$ was decreased over an 8-week period of pitavastatin therapy (Table 1). Consistent with previous research (de Groot et al., 1998; Smilde et al., 2001), regressions of IMT were presented in both Groups 1 and 2 (Group 1: the range from $(0.78 \pm 0.05)$ to $(0.75 \pm 0.05) \mathrm{mm}$; Group 2: from $(0.76 \pm 0.04)$ to $(0.74 \pm 0.05) \mathrm{mm})$, although no significant change of IMT was detected (Fig. 1). The dose or duration of pitavastatin may have been insufficient to confer a significant alteration of IMT in the HC patient. Nonetheless, the fact that regression of IMT, together with our previous research, suggests that these beneficial effects on inhibiting the progression of AS are mediated partly by lipid-lowering and, partly by the improvement of ED through the antioxidative property of pitavastatin calcium.

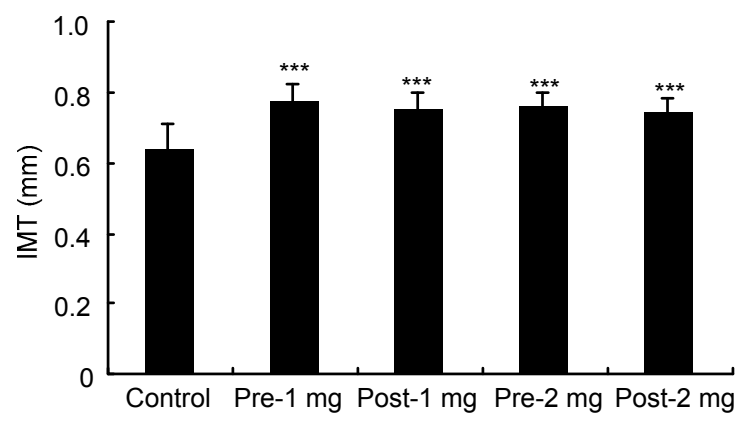

Fig. 1 Influences of dose-dependent pitavastatin calcium on IMT

${ }^{* * *} P<0.001$ vs. control

Potential limitations of this study are that the drug-exposure time is short, the follow-up period is short, and patient numbers are small; therefore, further study is required to identify specific data that contribute to the anti-atherosclerosis property of pitavastatin calcium in $\mathrm{HC}$ patients; results of further clinical studies on the pleiotropic effect of pitavastatin 
calcium are also needed. In spite of these limitations, however, we believe that pitavastatin calcium plays a crucial role in protecting arteries against the development of atherosclerosis in response to HC.

\section{Conclusions}

Treatment with pitavastatin calcium exerts favorable effects on endothelial function and arterial stiffness. It also ameliorated carotid atherosclerosis in patients with HC.

\section{Compliance with ethics guidelines}

Jing ZHAO, Hui-min YAN, Ya LI, Jia WANG, Lu HAN, Zhi-hao WANG, Meng-xiong TANG, Wei ZHANG, Yun ZHANG, and Ming ZHONG declare that they have no conflict of interest.

All procedures followed were in accordance with the ethical standards of the responsible committee on human experimentation (institutional and national) and with the Helsinki Declaration of 1975, as revised in 2008 (5). Informed consent was obtained from all patients for being included in the study. Additional informed consent was obtained from all patients for whom identifying information is included in this article.

\section{References}

Anderson, T.J., 2006. Arterial stiffness or endothelial dysfunction as a surrogate marker of vascular risk. Can. J. Cardiol., 22(Suppl. B):72B-80B. [doi:10.1016/S0828282X(06)70990-4]

Anderson, T.J., Uehata, A., Gerhard, M.D., et al., 1995. Close relation of endothelial function in the human coronary and peripheral circulations. J. Am. Coll. Cardiol., 26(5): 1235-1241. [doi:10.1016/0735-1097(95)00327-4]

Barter, P., Gotto, A.M., LaRosa, J.C., et al., 2007. HDL cholesterol, very low levels of LDL cholesterol, and cardiovascular events. N. Engl. J. Med., 357(13):1301-1310. [doi:10.1056/NEJMoa064278]

Bonetti, P.O., Lerman, L.O., Lerman, A., 2003. Endothelial dysfunction: a marker of atherosclerotic risk. Arterioscler Thromb. Vasc. Biol., 23(2):168-175. [doi:10.1161/01. ATV.0000051384.43104.FC]

Bots, M.L., Hoes, A.W., Koudstaal, P.J., et al., 1997. Common carotid intima-media thickness and risk of stroke and myocardial infarction: the Rotterdam Study. Circulation, 96(5):1432-1437. [doi:10.1161/01.CIR.96.5.1432]

Burke, G.L., Evans, G.W., Riley, W.A., et al., 1995. Arterial wall thickness is associated with prevalent cardiovascular disease in middle-aged adults: the Atherosclerosis Risk in Communities (ARIC) Study. Stroke, 26(3):386-391. [doi:10.1161/01.STR.26.3.386]

Chen, E.J., Adler, R.S., Carson, P.L., et al., 1995. Ultrasound tissue displacement imaging with application to breast cancer. Ultrasound Med. Biol., 21(9):1153-1162. [doi:10. 1016/0301-5629(95)02005-5]

Cho, S.H., Jeong, M.H., Park, I.H., et al., 2009. Endothelial dysfunction, increased carotid artery intima-media thickness and pulse wave velocity, and increased level of inflammatory markers are associated with variant angina. $J$. Cardiol., 54(2):183-191. [doi:10.1016/j.jjcc.2009.05.003]

Corretti, M.C., Anderson, T.J., Benjamin, E.J., et al., 2002. Guidelines for the ultrasound assessment of endothelialdependent flow-mediated vasodilation of the brachial artery: a report of the International Brachial Artery Reactivity Task Force. J. Am. Coll. Cardiol., 39(2):257-265. [doi:10.1016/S0735-1097(01)01746-6]

de Groot, E., Jukema, J.W., Montauban van Swijndregt, A.D., et al., 1998. B-mode ultrasound assessment of pravastatin treatment effect on carotid and femoral artery walls and its correlations with coronary arteriographic findings: a report of the Regression Growth Evaluation Statin Study (REGRESS). J. Am. Coll. Cardiol., 31(7):1561-1567. [doi:10.1016/S0735-1097(98)00170-3]

Dembowski, E., Davidson, M.H., 2009. A review of lipid management in primary and secondary prevention. $J$. Cardiopulm. Rehabil. Prev., 29(1):2-12. [doi:10.1097/ HCR.0b013e318192754e]

Diehm, C., Kareem, S., Lawall, H., 2004. Epidemiology of peripheral arterial disease. Vasa, 33(4):183-189. [doi:10. 1024/0301-1526.33.4.183]

Galle, J., Bassenge, E., Busse, R., 1990. Oxidised low density lipoproteins potentiate vasoconstrictions to various agonists by direct interaction with vascular smooth muscle. Circ. Res., 66(5):1287-1293. [doi:10.1161/01.RES.66.5. 1287]

Hiro, T., Kimura, T., Morimoto, T., et al., 2009. Effect of intensive statin therapy on regression of coronary atherosclerosis in patients with acute coronary syndrome: a multicenter randomized trial evaluated by volumetric intravascular ultrasound using pitavastatin versus atorvastatin (JAPAN-ACS Study). J. Am. Coll. Cardiol., 54(4): 293-302. [doi:10.1016/j.jacc.2009.04.033]

Juonala, M., Viikari, J.S., Laitinen, T., et al., 2004. Interrelations between brachial endothelial function and carotid intima-media thickness in young adults: the Cardiovascular Risk in Young Finns Study. Circulation, 110(18): 2918-2923. [doi:10.1161/01.CIR.0000147540.88559.00]

Leone, N., Ducimetière, P., Gariépy, J., et al., 2008. Distension of the carotid artery and risk of coronary events: the three-city study. Arterioscler. Thromb. Vasc. Biol., 28(7): 1392-1397. [doi:10.1161/ATVBAHA.108.164582]

Lorenz, M.W., Markus, H.S., Bots, M.L., et al., 2007. Prediction of clinical cardiovascular events with carotid intimamedia thickness: a systematic review and meta-analysis. Circulation, 115(4):459-467. [doi:10.1161/CIRCULATI ONAHA.106.628875]

Lusis, A.J., 2000. Atherosclerosis. Nature, 407(6801):233-241. [doi:10.1038/35025203] 
Makita, S., Ohira, A., Tachieda, R., et al., 2000. Dilation and reduced distensibility of carotid artery in patients with abdominal aortic aneurysms. Am. Heart J., 140(2):297302. [doi:10.1067/mhj.2000.108000]

Neunteufl, T., Katzenschlager, R., Hassan, A., et al., 1997. Systemic endothelial dysfunction is related to the extent and severity of coronary artery disease. Atherosclerosis, 129(1):111-118. [doi:10.1016/S0021-9150(96)06018-2]

Oliver, J.J., Webb, D.J., 2003. Noninvasive assessment of arterial stiffness and risk of atherosclerotic events. Arterioscler. Thromb. Vasc. Biol., 23(4):554-566. [doi:10. 1161/01.ATV.0000060460.52916.D6]

Petnehazy, T., Stokes, K.Y., Russell, J.M., et al., 2005. Angiotensin II type-1 receptor antagonism attenuates the inflammatory and thrombogenic responses to hypercholesterolemia in venules. Hypertension, 45(2):209-215. [doi:10.1161/01.HYP.0000154085.27868.93]

Raitakari, O.T., Juonala, M., Kähönen, M., et al., 2003. Cardiovascular risk factors in childhood and carotid artery intima-media thickness in adulthood: the Cardiovascular Risk in Young Finns Study. JAMA, 290(17):2277-2283. [doi:10.1001/jama.290.17.2277]

Ross, R., 1993. The pathogenesis of atherosclerosis: a perspective for the 1990s. Nature, 362(6423):801-809. [doi:10.1038/362801a0]

Ross, R., 1999. Atherosclerosis - an inflammatory disease. $N$. Engl. J. Med., 340(2):115-126. [doi:10.1056/NEJM1999 01143400207]

Serruys, P.W., de Feyter, P., Macaya, C., et al., 2002. Fluvastatin for prevention of cardiac events following successful first percutaneous coronary intervention: a randomized controlled trial. JAMA, 287(24):3215-3222. [doi:10.1001/ jama.287.24.3215]

Smilde, T.J., van Wissen, S., Wollersheim, H., et al., 2001. Effect of aggressive versus conventional lipid lowering on atherosclerosis progression in familial hypercholesterolaemia (ASAP): a prospective, randomised, doubleblind trial. Lancet, 357(9256):577-581. [doi:10.1016/ S0140-6736(00)04053-8]

Takase, B., Uehata, A., Akima, T., et al., 1998. Endotheliumdependent flow-mediated vasodilatation in coronary and brachial arteries in suspected coronary disease. Am. J. Cardiol., 82(12):1535-1539. [doi:10.1016/S0002-9149 (98)00702-4]

Weidner, G., 2000. Why do men get more heart disease than women? An international perspective. J. Am. Coll. Health., 48(6):291-294. [doi:10.1080/0744848000959627x0]

Yan, H.M., Zhao, J., Ma, D.Z., et al., 2011. The effect of pitavastatin calcium on endothelial dysfunction induced by hypercholesterolemia. Expert Opin. Pharmacother., 12(10):1463-1471. [doi:10.1517/14656566.2011.583238]

Yeboah, J., Crouse, J.R., Hsu, F.C., et al., 2007. Brachial flowmediated dilation predicts incident cardiovascular events in older adults: the Cardiovascular Health Study. Circulation, 115(18):2390-2397. [doi:10.1161/CIRCULATI ONAHA.106.678276]
Yu, X.Y., Zhao, Y., Song, X.X., et al., 2014. Association between non-alcoholic fatty liver disease and arterial stiffness in the non-obese, non-hypertensive, and nondiabetic young and middle-aged Chinese population. $J$. Zhejiang Univ.-Sci. B (Biomed. \& Biotechnol.), 15(10): 879-887. [doi:10.1631/jzus.B1400028]

Zanchetti, A., Bond, M.G., et al., Hennig, M., 2002. Calcium antagonist lacidipine slows down progression of asymptomatic carotid atherosclerosis: principal results of the European lacidipine study on atherosclerosis (ELISA), a randomized, double-blind, long-term trial. Circulation, 106(19):2422-2427. [doi:10.1161/01.CIR.0000039288. 86470.DD]

\section{中文概要}

\section{题 目: 匹伐他汀钙对高胆固醇血症患者动脉弹样硬化的 影响}

目 的: 观察匹伐他汀钻对高胆固醇血症患者外周血管的 影响。

创新点: 首次在国内发现匹伐他汀钲能够改善高胆固醇血 症患者肱动脉和颈动脉血管内皮功能而且延缓 其动脉籿样硬化发展, 并首次证实改善内皮功能 是匹伐他汀铂延缓其动脉粥样硬化发展的重要 原因。

方 法: 按照入选排除标准, 选取本院高胆固醇血症患者 ( $\mathrm{HC}$ ), 完成超声心动图检查的 40 例。根据剂 量不同, 分为两个剂量组: $1 \mathrm{mg}$ 剂量组 20 例 (男 性 5 例, 女性 15 例, 平均年龄 (55.20土8.35)岁), $2 \mathrm{mg}$ 剂量组 20 例 (男性 9 例, 女性 11 例, 平均 年龄(57.56 \pm 6.09$)$ 岁)。访视结束后完成超声心动 图检查的 HC 组 36 例, 两个剂量组分别有 2 人失 访。治疗后 $1 \mathrm{mg}$ 剂量组 18 例 (男性 3 例, 女性 15 例, 平均年龄 $(56.00 \pm 7.85)$ 岁), $2 \mathrm{mg}$ 剂量组 18 例(男性 7 例, 女性 11 例, 平均年龄 (57.79 16.46 ) 岁)。选择本院同期体检中心 30 例正常人作为 对照 (年龄和性别均与病例组匹配, 男性 14 例, 女性 16 例, 平均年龄 $(54.94 \pm 6.90)$ 岁)。所有研究 对象, 均经隔夜禁食 12 14 小时, 次日清晨抽取 空腹肘静脉血, 测定临床生化指标。采用 Sequia 512 彩色多普勒超声诊断仪, 应用高分辩率外周 血管超声技术, 检测 $\mathrm{HC}$ 治疗前后肱动脉血流介 导性舒张功能 (FMD) 、颈动脉结构和功能。

结 论: 经匹伐他汀钻治疗 8 周后, 高胆固醇血症患者血 管功能明显改善, 表现为 FMD 升高, 僵硬度减 小; 颈动脉僵硬度和内中膜厚度 (IMT) 延缓进 展与其内皮功能改善密切相关。

关键词: 高胆固醇血症; 内皮功能; 颈动脉; 匹伐他汀钲 